\title{
Reflets
}

Revue d'intervention sociale et communautaire

\section{Faire basculer le climat de profilage social à Ottawa : le Programme de contestation des contraventions}

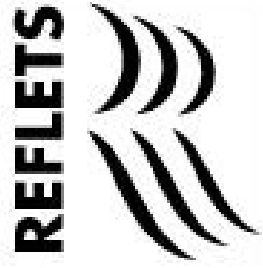

\section{Suzanne Bouclin}

Volume 22, numéro 1, printemps 2016

Profilage social et judiciarisation de la marginalité

URI : https://id.erudit.org/iderudit/1037165ar

DOI : https://doi.org/10.7202/1037165ar

Aller au sommaire du numéro

Éditeur(s)

Reflets, Revue d'intervention sociale et communautaire

ISSN

1203-4576 (imprimé)

1712-8498 (numérique)

Découvrir la revue

Citer cet article

Bouclin, S. (2016). Faire basculer le climat de profilage social à Ottawa : le Programme de contestation des contraventions. Reflets, 22(1), 123-157.

https://doi.org/10.7202/1037165ar
Résumé de l'article

Dans la région d'Ottawa, le Programme de contestation des contraventions (ci-après appelé le PCC) offre des services de représentation juridique aux personnes en situation d'itinérance qui ont censément commis des infractions réglementaires. Les personnes qui se prévalent du PCC subissent du profilage social sous la forme d'avis d'infraction émis en vertu de diverses lois provinciales et de divers règlements municipaux, notamment la Loi de 1999 sur la sécurité dans les rues, pour des motifs reliés à l'occupation de l'espace public. Même si le PCC a eu du succès en aidant les personnes utilisatrices à régler ces procès-verbaux de contravention (aussi communément appelé « avis d'infraction » ou " contravention "), l'entrée en vigueur de la Loi sur l'accès à la justice de l'Ontario a forcé le PCC à cesser ses activités. Cependant, à l'automne 2014, le programme a été relancé à la Section de common law de la Faculté de droit, de l'Université d'Ottawa. La première partie du présent article trace un profil démographique de l'itinérance à Ottawa dans une optique critique de la judiciarisation de l'itinérance. La deuxième présente un aperçu de la structure et du travail du PCC dans sa première mouture de 2003 à 2007. La troisième donne une description de sa structure depuis 2014 et dresse un bilan des diverses dispositions réglementaires et législatives en vertu desquelles les utilisatrices et utilisateurs des services du PCC continuent de se voir imposer des contraventions. Enfin, la conclusion dégage certains des défis particuliers auxquels le PCC fait toujours face en s'attaquant au manque d'accès à la justice pour les personnes en situation d'itinérance.
Tous droits réservés @ Reflets, Revue d’intervention sociale et communautaire, 2016
Ce document est protégé par la loi sur le droit d'auteur. L'utilisation des services d'Érudit (y compris la reproduction) est assujettie à sa politique d'utilisation que vous pouvez consulter en ligne. 


\title{
Faire basculer le climat de profilage social à Ottawa : le Programme de contestation des contraventions
}

\author{
Suzanne Bouclin \\ Professeure agrégée, Faculté de droit, Université d'Ottawa
}

\section{Résumé}

Dans la région d'Ottawa, le Programme de contestation des contraventions $^{1}$ (ci-après appelé le PCC) offre des services de représentation juridique aux personnes en situation d'itinérance qui ont censément commis des infractions réglementaires. Les personnes qui se prévalent du PCC subissent du profilage social sous la forme d'avis d'infraction émis en vertu de diverses lois provinciales et de divers règlements municipaux, notamment la Loi de 1999 sur la sécurité dans les rues, pour des motifs reliés à l'occupation de l'espace public. Même si le PCC a eu du succès en aidant les personnes utilisatrices à régler ces procès-verbaux de contravention (aussi communément appelé "avis d'infraction » ou " contravention "), l'entrée en vigueur de la Loi sur l'accès à la justice de l'Ontario a forcé le PCC à cesser ses activités. Cependant, à l'automne 2014, le programme a été relancé à la Section de common law de la Faculté de droit, de l'Université d'Ottawa. La première partie du présent article trace un profil démographique de l'itinérance à Ottawa dans une optique critique de la judiciarisation de l'itinérance. La deuxième présente un aperçu de la structure et du travail du PCC dans sa première 
mouture de 2003 à 2007. La troisième donne une description de sa structure depuis 2014 et dresse un bilan des diverses dispositions réglementaires et législatives en vertu desquelles les utilisatrices et utilisateurs des services du PCC continuent de se voir imposer des contraventions. Enfin, la conclusion dégage certains des défis particuliers auxquels le PCC fait toujours face en s'attaquant au manque d'accès à la justice pour les personnes en situation d'itinérance.

Mots clés : accès à la justice, loi sur la sécurité dans les rues, profilage social, judiciarisation de l'itinérance

\section{Abstract}

The Ticket Defence Program (TDP) has since 2003 offered free representation to street-involved people experiencing social profiling in the Ottawa region. Its service users are homeless or street-involved people who have received provincial and municipal offences for income-generation activities such as panhandling as well as other activities associated with life on the street. While forced to cease operations as a result of new regulations around the provision of legal services in Ontario,TDP re-emerged as the Programme de contestation des contraventions in 2014 as a bilingual, campus-community partnership offering free legal services and representation for provincial and municipal offences. Part one of this research provides an overview of the heterogeneous population that is street-involved in Ottawa through a lens of the criminalization of poverty. Part two describes TDP's original structure between 2003 and 2007. Part three details some of the changes it underwent to meet Law Society of Upper Canada's requirements around allowable legal practice and lays out some of the most common charges the PCC has seen over the last few years. The article concludes with a discussion of the on-going challenges this mobile community initiative faces in its efforts to provide meaningful access to justice.

Key words: access to justice, street-involved people, social profiling, criminalization of poverty 


\section{Introduction}

Dans plusieurs grandes villes du Canada, les personnes en situation d'itinérance font l'objet de stigmatisation, de harcèlement et d'intimidation de la part des services policiers, qui veulent ainsi dissiper un présumé sentiment d'insécurité des citoyennes et citoyens dans le cadre de leurs activités quotidiennes dans des lieux publics.

La présence des personnes itinérantes est régulée dans certains secteurs géographiques des grandes villes afin d'en préserver l'esthétique, de donner suite aux plaintes des commerçants et de se débarrasser des personnes "dérangeantes » sur la place publique, avant l'arrivée saisonnière des touristes. Les administrations municipales et les gouvernements provinciaux cherchent à éliminer la présence des personnes itinérantes de l'espace public en ayant recours à des mesures de déplacement et d'expulsion et, surtout, à des dispositions réglementaires (non pénales) qui sont objectives en apparence, mais qui ciblent les personnes en situation d'itinérance (FEANTSA, 2015; Sylvestre, et collab., 2011).

Ces lois gèrent les « incivilités » (manquement aux règlements municipaux sur le bruit, l'usage d'alcool ou de tabac, ou manquement aux lois provinciales, comme le fait de quémander) et définissent des catégories de comportement (surtout celles liées aux activités rémunératrices des personnes itinérantes) pour lesquelles une intervention policière serait légitime (FEANTSA, 2015; Bellot, et collab., 2007; Pichon, 2009; Blomley, 2010; Sylvestre, et collab., 2011; Sylvestre, 2012; Wacquant, 2012; Bouclin, 2014).

En 2009, au Québec, la Commission des droits de la personne et des droits de la jeunesse a qualifié cette répression de profilage social et l'a assimilée à une forme de discrimination (Campbell et Eid, 2009). Ce n'est pas le cas en Ontario, où les agents des infractions provinciales ${ }^{2}$ possèdent un outil de coercition important, soit la Loi sur les infractions provinciales de l'Ontario (ciaprès «LIP ") (1990), qui leur confere un pouvoir d'arrestation 
"Depuis 2003, le Ticket Defence Program, ciaprès appelé "TDP », offre de l'accompagnement ou de la représentation juridique aux personnes itinérantes de la région d'Ottawa qui ont reçu de telles contraventions. » lorsqu'ils croient, en se fondant sur des motifs raisonnables, qu'un individu a violé une disposition d'une loi provinciale ou d'un règlement municipal (art. 145). La présente recherche situe donc les règlements provinciaux et municipaux de cette province et leur application dans l'optique de la pénalisation et de la judiciarisation de la pauvreté (Wacquant, 2012; Poupart, 2004; Goffman, 2014), soit le recours à des discours, à des pratiques et à des outils juridiques pour gérer les problèmes sociaux liés aux inégalités socioéconomiques.

Depuis 2003, le Ticket Defence Program, ciaprès appelé "TDP ", offre de l'accompagnement ou de la représentation juridique aux personnes itinérantes de la région d'Ottawa qui ont reçu de telles contraventions. Dans un premier temps, le présent article fournit un profil démographique de l'itinérance à Ottawa. Dans un deuxième temps, il propose un tour d'horizon de la structure et du travail du TDP jusqu'en 2007. Dans un troisième temps, l'article donne une description de la nouvelle structure du programme, connu sous le nom de Programme de contestation des contraventions (ci-après appelé "PCC») depuis 2014, et dresse un bilan des diverses dispositions réglementaires et législatives en vertu desquelles les personnes qui se prévalent du PCC se voient actuellement décerner des contraventions. Finalement, nous soulignons certains des défis auxquels le PCC fait toujours face en s'efforçant de faciliter l'accès à la justice pour les personnes en situation d'itinérance.

\section{I) Profil démographique de l'itinérance à Ottawa}

L'itinérance est un phénomène et problème social « qui ne peut se résumer ni s'expliquer par une formule simple " (Roy et Hurtubise, 2007, p. 1). Les personnes en situation d'itinérance forment un groupe hétérogène faisant face à des problèmes socioéconomiques comme la pauvreté extrême, le chômage ou le sous-emploi, l'insuffisance des programmes de santé ou des programmes sociaux, le manque d'accès aux programmes de réduction des risques ou de traitement de la toxicomanie, 
et l'absence de logement stable et convenable. À ces difficultés s'ajoutent souvent des expériences personnelles de violence, de stress post-traumatique, de racisme, de traumatisme intergénérationnel, de problème de jeu ou d'endettement, ainsi que l'existence d'un cycle de criminalisation, d'incarcération/ institutionnalisation et de libération/ désinstitutionnalisation, sans aide sociale appropriée. Nous explorons ici trois dynamiques du phénomène de l'itinérance à Ottawa : l'itinérance absolue, l'itinérance cachée et la surreprésentation des personnes d'origine autochtone en situation d'itinérance. Nous abordons ensuite l'optique dans laquelle s'inscrit la présente recherche : la judiciarisation de l'itinérance.

Jusqu'à récemment, la littérature dominante sur l'itinérance ciblait dans une large mesure les personnes vivant une situation d'itinérance absolue, visible et chronique (Brown et Capponi, 1993; Centre national d'information sur la violence dans la famille, 2006, ci-après Novac, 2006). Cette catégorie, qui renvoie à l'absence de domicile, vise les personnes qui dorment dans des endroits non désignés pour cette utilisation, comme la rue, les parcs ou le dessous des ponts (Comité des sans-abri, 1987).

En 2002, une recherche a révélé que $61 \%$ des Ottaviens en situation d'itinérance absolue n'utilisaient les refuges d'urgence

"Une étude menée par l'Alliance pour mettre un terme à l'itinérance à

Ottawa... (2009) indique qu'en 2008 et 2009, il y a eu 7445 demandes pour un lit dans un refuge, dont 3570 demandes émanant de personnes s'identifiant comme des hommes d'âge adulte." qu'en dernier ressort (Farrell, Aubry et Reissing, 2001). En 2009, selon la Ville d'Ottawa, il y a eu, chaque soir, entre 16 et 45 personnes dans la rue qui ont dû dormir à l'extérieur. Cependant, l'itinérance visible fait aussi référence aux personnes qui utilisent des ressources d'hébergement. Une étude menée par l'Alliance pour mettre un terme à l'itinérance à Ottawa (Alliance to End Homelessness Ottawa, ci-après "ATEHO ") (2009) indique qu'en 2008 et 2009, il y a eu 7445 demandes pour un lit dans un refuge, dont 3570 demandes émanant de personnes s'identifiant comme des hommes d'âge adulte.

En dépit de la perception que « le terme itinérant est l'appellation consacrée pour parler globalement des "hommes à la rue" " (Roy, 2009, p. 1), plusieurs femmes dorment, à Ottawa, dans des parcs, dans des escaliers d'édifices, sur des trottoirs et aussi dans des édifices abandonnés (squats) (Novac, 2002). Czapska, et collab. 
«En 2013, le rapport sur l'état de l'itinérance au Canada a évalué l'itinérance cachée pour cette même année à 50000 personnes au cours d'une nuit donnée au pays..."
(2008) notent aussi une hausse des femmes dans les refuges à Ottawa dans une proportion de $14,5 \%$ de 2005 à 2008. Selon l'ATEHO, en 2009, les femmes célibataires adultes ont eu recours à des refuges 994 fois à Ottawa (2009).

Il n'y a pas seulement les femmes qui deviennent plus nombreuses dans la population itinérante. Dans le même bilan, l'ATEHO (2009) estime que 775 demandes pour un refuge ont été remplies par des familles, 1317 par des enfants ou des jeunes de moins de 16 ans, et 411 par des jeunes de moins de 18 ans. Qui plus est, en 2014, l'itinérance des jeunes âgés de 16 à 25 ans a augmenté de $24 \%$ à Ottawa (ATEHO, 2015). Toujours selon l'ATEHO, en 2014, les jeunes constituaient $30 \%$ des personnes itinérantes à Ottawa, et $23 \%$ de ceux-ci étaient d'origine autochtone (ATEHO, 2015). La moitié des jeunes en situation d'itinérance à Ottawa s'identifient comme lesbiennes, gais, bisexuels, transgenres ou bispirituels (Kirkup, 2013). Molnar, et collab. (1998) affirment que les jeunes itinérants de sexe masculin de la région d'Ottawa font état d'un niveau de détresse psychologique deux fois plus élevé que celui de leurs homologues non itinérants. Selon Kauppi, et collab. (2012), les francophones ont des caractéristiques spécifiques qui les rendent plus vulnérables à l'itinérance, notamment le fait de faire partie d'un groupe minoritaire. Quant aux jeunes francophones en situation d'itinérance dans la capitale nationale, ils auront, selon Molgat (1995), "tendance à se construire une identité bilingue ou anglophone afin d'éviter un plus grand isolement » (p. 146).

Une deuxième catégorie d'itinérance est celle qui est "cachée " et souvent vécue par des personnes et des familles hébergées de façon temporaire ou provisoire chez des connaissances ou des membres de la famille ou installées dans leur voiture. En 2013, le rapport sur l'état de l'itinérance au Canada a évalué l'itinérance cachée pour cette même année à 50000 personnes au cours d'une nuit donnée au pays (Gaetz, et collab., 2013). S'ajoute à cette catégorie celle de l'itinérance relative, vécue par des personnes et des familles qui sont sans protection suffisante contre les éléments, sans accès à de l'eau potable et à des installations sanitaires, ou sans logement stable, ou dans des situations où la sécurité personnelle ou financière et /ou l'accès aux soins de santé sont précaires 
(Novac, 2006). L'itinérance cachée et l'itinérance relative sont particulièrement présentes chez les femmes, surtout les femmes autochtones (Walsh, et collab., 2011; Amnistie internationale, 2009 , p. 11), ou chez les survivantes de violence entre partenaires intimes (Lenon, 2000).

La Maison d'amitié, un centre d'hébergement d'Ottawa qui vient en aide aux survivantes de violence entre partenaires intimes et à leurs enfants, estime qu'en 2005 et 2006 près de 106000 personnes ont eu recours aux services de refuge du genre au Canada (Maison d'amitié, 2013; Echenberg et Jensen, 2009). Faute d'espace, la Maison d'amitié d'Ottawa a été obligée de refuser 937 demandes d'hébergement en 2004 avant de procéder à l'ouverture d'un deuxième centre en 2006 (Maison d'amitié, 2013).

L'importante surreprésentation des personnes d'origine autochtone en situation d'itinérance à Ottawa, comme dans toutes les grandes villes du Canada, ne peut être surestimée (Klos, 1997; Regroupement des centres d'amitié autochtones du Québec, 2008; Ship, 2001). Toutefois, il y a un manque de données en ce qui a trait à cette population en situation d'itinérance. Néanmoins, on estime qu'en Ontario, les personnes autochtones représentent entre $20 \%$ et $30 \%$ de la population itinérante (Belanger, et collab., 2013), alors qu'elles ne représentent que 1,3\% de la population provinciale. Selon le recensement de 2006, 20590 personnes d'origine autochtone nord-américaine vivaient dans la région de la capitale nationale (Germain, Costa et Kelly-Scott, 2009). En 2008, les personnes de cette même population constituaient $1,6 \%$ de la population d'Ottawa (Germain, et collab., 2009). Une enquête menée auprès des personnes itinérantes d'Ottawa a révélé qu'en 2002 et 2003,17\% des répondantes et répondants étaient d'origine autochtone (Association des étudiant(e)s infirmier(ère)s du Canada, 2014).Afin de comprendre cette réalité, il est nécessaire de voir le phénomène de l'itinérance sous un angle post-colonialiste, ce qui est souvent omis dans les définitions plus orthodoxes de l'itinérance (soit absolue ou invisible). Comme l'indique l'Assemblée des Premières Nations du Québec et du Labrador (APNQL) : 
[L]'itinérance, chez les Premières Nations, est directement liée aux conséquences sociales et psychologiques des pensionnats autochtones, telles que la perte du sens de la vie, la perte de repères socioculturels, la perte de la langue et le vide identitaire créé par les tentatives répétées d'assimiler les Premières Nations en tuant "l'Indien dans l'enfant ». Parmi les causes de l'itinérance - dont certaines sont étroitement liées aux conséquences du développement des écoles résidentielles - [les recherches révèlent] le manque de logement abordable, la pauvreté, des problèmes de santé mentale et physique, les dépendances, la violence familiale, le phénomène migratoire, la marginalisation d'un membre par sa communauté et certaines lacunes dans les services de santé assurés par Santé Canada. (Sider, 2005, cité dans APNQL, 2008, p. 20-21)

Il importe de souligner qu'à Ottawa, malgré l'existence de nombreux fournisseurs de services autochtones d'excellente qualité, les personnes autochtones en situation d'itinérance éprouvent toujours de grandes difficultés à trouver des services adaptés à leur culture respective lorsqu'elles fréquentent des refuges non autochtones (mainstream agencies); ces derniers requièrent toujours une meilleure sensibilisation aux effets de l'impérialisme culturel et du racisme systémique sur la collectivité autochtone (Social Data Research Ltd., 2005, citant Webster, 2007; Kauppi, et collab., 2012).

\section{Judiciarisation de l'itinérance}

Malgré des différences importantes en ce qui a trait aux expériences individuelles, au trajet et au vécu des personnes en situation d'itinérance, nombre de recherches font état de quelques points communs : les personnes en situation d'itinérance font face à de la stigmatisation et à de l'insécurité de vivre dans les rues. De plus, il y a une intensification de la judiciarisation des 
"Malgré des

différences importantes

en ce qui a trait

aux expériences

individuelles, au

trajet et au vécu des

personnes en situation

d'itinérance, nombre

de recherches font

état de quelques

points communs: les

personnes en situation

d'itinérance font face

à de la stigmatisation et à de l'insécurité de vivre dans les rues." personnes itinérantes sous forme de " dispositions réglementaires et législatives relatives à l'utilisation du domaine public, ainsi que dans la manière dont ces instruments juridiques sont appliqués par les forces policières " (Campbell et Eid, 2009, p. 2; voir aussi Bernier, et collab., 2011; Bouclin, 2014).

$\mathrm{La}$ judiciarisation réfere aux processus et aux pratiques qui privilégient le recours aux institutions juridiques officielles (tels les tribunaux) pour régler des différends qui " pourraient être réglés par d'autres voies (médiation, accord amiable) ». Elle réfere également à l'" intervention croissante des juges dans le contrôle de la régularité des actes de certaines autorités (élus, administrateurs, chefs d'entreprise, etc.) " (Bouclin et Sala, 2013,p. 6). Selon Bernier, et collab. (2011), la judiciarisation des personnes itinérantes « s'inscrit dans le contexte de l'adoption de politiques municipales de luttes aux incivilités dans les années 1990 " et s'inspire de la théorie du carreau brisé (Broken Window Theory) (2011, p. 5).

En Ontario, l'adoption de la Loi de 1999 sur la sécurité dans les rues (ci-après appelée la " $L S R$ ») est un exemple de cette théorie mise en pratique. La LSR tombe dans la catégorie des dispositions législatives générales sur l'ordre public et la sécurités. Elle a créé plusieurs nouvelles infractions provinciales et proscrit également certaines formes de mendicité, qu'elle considère comme de la sollicitation "agressive " qui "inquiétera vraisemblablement une personne raisonnable quant à sa sécurité » (par. 2(1)). Est également interdite la sollicitation d'une personne retenue dans certains lieux publics, par exemple une personne qui attend l'autobus (al. $3(2) c))$. La LSR interdit aussi les activités commerciales auprès des automobilistes, par exemple s'adonner au "squeegee ", c'està-dire s'approcher des véhicules immobilisés sur la route, surtout au feu rouge, et offrir de laver les pare-brise en espérant recevoir en retour de l'argent des conducteurs (al. 3(2)f $f)$ ).

La LSR a eu comme conséquence directe d'augmenter la répression envers les personnes en situation d'itinérance à Toronto (Esmonde, 2002; Hermer et Mosher, 2002; Collins et Blomley, 2003) et dans la région de la capitale nationale (Sylvestre, et collab., 2011; Bouclin, 2014). Des statistiques obtenues en vertu d'une 
"Cependant, l'effet éducatif ou même dissuasif de l'application de la loi demeure quelque peu insaisissable. " demande d'accès à l'information démontrent que, du $1^{\text {er }}$ janvier 2010 au 22 septembre 2014, les agents de police d'Ottawa ont établi pas moins de 6921 procès-verbaux de contravention (aussi communément appelés " avis d'infraction " ou " contraventions ") en vertu de la $L S R^{4}$. La majorité de ces procès-verbaux a été dressée au marché By et au centre-ville d'Ottawa (SPO, 2014). Qui plus est, à Ottawa, les forces de l'ordre se sont dotées d'autres outils législatifs répressifs, uniques à la région de la capitale nationale, en vertu des règlements de la Commission de la capitale nationale $(\mathrm{CCN})$.

D'après les données publiées sur leur site Web, la CCN et le gouvernement fédéral possèdent et gèrent 4500 hectares de terrains, surtout des parcs, des promenades panoramiques et des sentiers, à Ottawa et à Gatineau (CCN-NCC, 2012). Considérés comme ayant une importance stratégique et symbolique pour la capitale nationale, puisqu'ils offrent aux habitants et aux touristes des endroits uniques, ces terrains sont surveillés de près afin de marier nature et culture. Le marché By fait partie des terrains de la CCN et, en vertu de sa loi habilitante, la Loi sur la capitale nationale ( $L C N »)$, la $C C N$ peut créer et adopter des règlements pour protéger ses propriétés et maintenir l'ordre.

Ces deux lois, la $L S R$ et la $L C N$, constituent d'importants outils législatifs employés par le Service de police d'Ottawa (SPO) pour répondre aux pressions exercées par la Zone d'améliorations commerciales du marché By (Byward Market Business Improvement Area) dans le but de s'occuper des personnes de la rue qui sont susceptibles de porter atteinte aux transactions commerciales (Seymour, 2015). Dans son rapport annuel de 2013, le SPO soutient que les incivilités, dont la sollicitation, nuisent à la "jouissance sûre et licite des rues, des trottoirs et des parcs du centre-ville ». La stratégie "à l'égard des contrevenants » du SPO est, selon l'inspecteur Chris Rhéaume, "l'éducation, par le truchement de l'application des règlements" (SPO, 2013, p. 11).

Cependant, l'effet éducatif ou même dissuasif de l'application de la loi demeure quelque peu insaisissable. L'interdiction de la plupart des sollicitations complique et restreint les activités 
génératrices de revenu pour des personnes déjà fragilisées par une précarité économique, par des problèmes de santé mentale ou de dépendance ou par la "précarité constante " (Laberge, Poirier et Charest, 1998, p. 20) qui frappe les personnes itinérantes; et ce n'est là qu'un des facteurs multidimensionnels qui influencent leur vie de tous les jours. Les personnes en situation d'itinérance finissent généralement par ignorer ces contraventions, ce qui entraîne en soi d'autres répercussions négatives. Une amende impayée sans déclaration d'intention de la contester est assimilée à une déclaration de culpabilité automatique, en vertu de la Loi sur les infractions provinciales de l'Ontario (art. 66.1 de la LIP), qui régit le processus de poursuite et d'exécution des infractions réglementaires provinciales et municipales. Cette loi prévoit qu'une amende doit être payée dans les 15 jours suivant son imposition, sinon il y a défaut de paiement (art. 69 de la LIP). À cette amende s'ajoutent les frais de justice et la suramende compensatoire pour l'aide aux victimes (Cour de justice de l'Ontario, 2016). Les personnes en situation d'itinérance omettent souvent, sinon toujours, de payer l'amende après l'inscription automatique de culpabilité, soit par manque d'argent, par ignorance du procès-verbal de contravention, ou par indifférence (Bouclin, 2014). Leur dette juridique est donc transmise à une agence d'évaluation du crédit et à une agence de recouvrement (Cour de justice de l'Ontario, 2016). Cette situation fait en sorte que de nombreuses personnes itinérantes accumulent des dettes considérables, pouvant s'élever à plusieurs milliers de dollars ${ }^{5}$, et que toute démarche exigeant une vérification de solvabilité, comme obtenir un logement, un

"C'est dans ce contexte de répression et d'injustice qu'en 2003 quelques citoyennes et citoyens indignés se sont réunis pour dénoncer le profilage social des Ottaviens en situation d'itinérance. » emploi, un permis de conduire ou une carte d'assurance-maladie, effectuer un retour aux études ou même se procurer des services de base comme le téléphone ou l'électricité, puisse éventuellement entraîner un refus (Saelinger, 2006; Glowinski, 2009; Chesnay, Bellot et Sylvestre, 2013; Bouclin, 2014).

C'est dans ce contexte de répression et d'injustice qu'en 2003 quelques citoyennes et citoyens indignés se sont réunis pour dénoncer le profilage social des Ottaviens en situation d'itinérance. Ils ont formé le TDP, nom qui a été traduit en 2014 par Programme de contestation des contraventions (PCC). 


\section{II) Aperçu du travail du PCC de 2003 à 2007}

Dans sa configuration d'origine, le TDP était composé de quatre catégories de bénévoles. D’abord, les «intervenants " étaient des intervenantes ou intervenants de rue conscients de la situation particulière des personnes itinérantes avec qui ils avaient déjà établi un rapport de confiance; ils prenaient les procès-verbaux d'infraction directement de leurs clients lors de leurs interactions quotidiennes dans les refuges. Si la personne avait son avis d'infraction en main, l'intervenante ou intervenant l'aidait à comprendre les trois options qui s'offraient à elle en vertu de la Loi sur les infractions provinciales. Selon cette loi, une personne peut, tout d'abord, plaider coupable en payant le montant total indiqué dans l'avis d'infraction (art. 8). Elle peut également se rendre au greffe du tribunal en vue d'une résolution rapide, en plaidant coupable et en présentant au juge de paix des explications et des observations quant à la peine, afin de faire modifier la sanction imposée (une amende réduite) ou de demander une prorogation du délai dans lequel l'amende doit être payée (art. 5). Enfin, elle peut plaider non coupable en remplissant un avis d'intention de comparaitre et demander la tenue d'un procès (art. 46). Une fois qu'un agent des infractions provinciales lui a signifié un avis d'infraction, la personne dispose de 15 jours pour choisir l'une de ces trois options, sinon elle sera considérée comme ne voulant pas contester l'accusation. Le juge de paix inscrira ensuite une déclaration de culpabilité au nom de la personne (Cour de justice de l'Ontario, 2016).

Aussitôt qu'une personne en situation d'itinérance recevait une contravention et en informait son intervenante ou intervenant, cette personne faisait alors toutes les démarches raisonnables pour recueillir et conserver l'avis d'infraction, faire signer un formulaire d'autorisation qui permettait aux bénévoles du TDP d'agir en son nom (dans ce cas, la personne en situation d'itinérance pouvait, si elle le souhaitait, être présente devant le tribunal, mais elle n'y était pas tenue), et d'aider l'utilisatrice ou l'utilisateur du service à remplir la documentation nécessaire en fonction 
de son choix quant à la façon de procéder (c'est-à-dire selon sa décision de plaider coupable ou non coupable). Les intervenantes et intervenants n'offraient pas de conseils juridiques ni de services de représentation devant le tribunal. Ces personnes transmettaient les documents et l'information à une deuxième catégorie de bénévoles, soit les " agentes ou agents » du TDP. Avant la mise en application de la Loi de 2006 sur l'accès à la justice, une personne qui faisait face à une poursuite pour une infraction provinciale ou municipale ("le défendeur ou la défenderesse ») pouvait comparaitre elle-même devant un juge de paix de la Cour de justice de l'Ontario ${ }^{6}$ ou par l'entremise d'une « représentante » ou d'un "représentant ", à savoir une personne autre qu'une avocate ou un avocat titulaire d'un permis du Barreau du Haut-Canada ${ }^{7}$. Appartenaient à cette catégorie, entre autres, les parajuristes (qui étaient auto-réglementés), une amie ou un ami, un membre de la famille non rémunéré, une employée ou un employé, ou un bénévole d'un syndicat. Les agentes et agents du TDP n'étaient pas des avocates, avocats ou parajuristes, mais plutôt des militantes et militants qui se sont organisés pour combattre les lois causant une injustice aux personnes en situation d'itinérance. Les agentes et agents agissaient au nom des personnes utilisatrices des services du TDP et, surtout, rencontraient la poursuivante ou le poursuivant (prosecutor) avant le jour du procès pour négocier des moyens possibles de régler l'affaire.

Une troisième catégorie de bénévoles offrait du soutien administratif et jouait essentiellement le rôle d'adjointe ou d'adjoint juridique : ces personnes présentaient systématiquement

«Entre 2003 et 2007, le TDP a permis d'atténuer les effets négatifs du profilage social exercé par le Service de police d'Ottawa (SPO) à l'endroit des personnes en situation d'itinérance. » des demandes de divulgation de la preuve (comme les notes des agents de police) pour chaque contravention recueillie; une autre inscrivait toutes les dates d'audience dans une base de données électronique et faisait transmettre les dates de comparution aux agentes et agents. La quatrième catégorie était celle des avocates et avocats bénévoles auxquels les agentes et agents pouvaient demander des conseils juridiques et obtenir des explications au sujet de la procédure judiciaire.

Entre 2003 et 2007, le TDP a permis d'atténuer les effets négatifs du profilage social exercé par le Service de police 
"Généralement, ces interventions ont entraîné une réduction considérable $d u$ montant de la peine ou une condamnation avec sursis..."
d'Ottawa (SPO) à l'endroit des personnes en situation d'itinérance. Le programme a recueilli environ 1200 avis d'infraction qui avaient été délivrés à des personnes en situation d'itinérance et dont la majorité se rapportait à des infractions reliées à la $L S R$, comme la sollicitation près des abribus, des guichets automatiques ou d'un véhicule sur la chaussée, et la sollicitation agressive (Bouclin, 2014).

La majorité de ces avis d'infraction a été traitée par les agentes et agents du TDP avant le procès. Le règlement le plus fréquent était un retrait de l'accusation par la poursuivante ou le poursuivant pour des raisons techniques (l'avis d'infraction mentionnait une disposition législative inexacte) ou par manque de preuve (le policier ou la personne responsable de l'application du règlement n'avaient pas suffisamment de notes ou n'étaient pas présents pour témoigner) (Bouclin, 2014). En fait, les agentes et agents du TDP n'ont eu à plaider devant la cour des infractions provinciales que dans un nombre restreint de cas; dans la plupart des cas, ils ont plaidé non coupables et ont eu gain de cause (Bouclin, 2014). Dans un petit nombre de cas, les agentes et agents du TDP ont eu à plaider coupables devant la cour des infractions provinciales; ils invoquaient des circonstances atténuantes pour leurs clients (pauvreté extrême, problèmes de santé mentale, toxicomanie ou dépendance grave, etc.). Généralement, ces interventions ont entraîné une réduction considérable du montant de la peine ou une condamnation avec sursis (Bouclin, 2014). Dans ce dernier cas, les agentes et agents tentaient de négocier des conditions raisonnables compte tenu de la situation des personnes utilisatrices et d'éviter des conditions qui pourraient fragiliser davantage leurs clientes et clients et entraîner un bris de conditions.

Toutefois, en 2007, le TDP a dû cesser ses activités à la suite de l'adoption, par le gouvernement ontarien, de la Loi de 2006 sur l'accès à la justice (LAJ). Celle-ci modifie la Loi sur le Barreau de l'Ontario ( $L B O)$ en stipulant que, désormais, les services de représentation juridique offerts par les parajuristes en Ontario seront réglementés par le Barreau du Haut-Canada. Désormais, il est interdit à tout parajuriste qui n'est pas titulaire d'un permis délivré par le Barreau d'exercer le droit (art. 26.1 de la LBO). 
Pour obtenir un tel permis, il faut être diplômé d'un programme de services juridiques agréé par le Barreau et satisfaire aux exigences relatives aux compétences professionnelles (art. 27 de la $L B O)$. Mais, quelques exceptions s'appliquent. Une personne qui fournit des services au sein d'un organisme sans but lucratif (par exemple une étudiante ou un étudiant en droit qui est bénévole ou une personne qui travaille dans une clinique d'Aide juridique Ontario) peut être exemptée de l'obligation d'être titulaire d'un permis (par. 21(5) de la $L B O$, règlements administratifs $\mathrm{n}^{\circ} 4$ et $\mathrm{n}^{\circ} 7.1$ de la $\left.L B O\right)$.

Bref, les agentes et agents du TDP offraient une représentation juridique nécessitant les compétences minimales d'un parajuriste titulaire d'un permis, mais n'appartenaient pas à une des catégories exemptées. Qui plus est, le Barreau avait des craintes quant à sa structure, notamment : les agentes et agents qui se présentaient devant la Cour de justice de l'Ontario n'étaient pas nécessairement les mêmes personnes que celles qui avaient effectué l'entrevue initiale. Selon le Barreau, cette structure allait à l'encontre des exigences concernant l'identification des clients, la vérification de l'identité des clients et la conservation de documents (Règlement administratif $n^{\circ} 7.1$ du Barreau du Haut-Canada; Bouclin, 2014, p. 63). Pour ces raisons, la demande du TDP afin de bénéficier d'une exemption au règlement a été rejetée à trois reprises (Bouclin, 2014). Par conséquent, les ambitions du TDP ont été contrecarrées de 2009 à 2014.

\section{III) Renaissance du Programme de contestation des contraventions de l'Université d'Ottawa}

Depuis ces événements, les mois se sont succédé et le profilage social des personnes en situation d'itinérance à Ottawa a perduré. Une professeure de droit de l'Université d'Ottawa a donc décidé de relancer le TDP suivant une formule qui serait conforme aux exigences du Barreau du Haut-Canada (Seymour, 2015).

À l'été 2014, le TDP a été remis sur pied selon une nouvelle formule. D'abord, alors que le programme n'était offert qu'en 
anglais, il a été développé en français, sous l'appellation de Programme de contestation des contraventions (PCC), puisque ses bénévoles offriraient désormais des services juridiques en français et en anglais. Une minorité faible, mais significative de ses utilisatrices et utilisateurs ont eu recours à la prestation de services en français.

De plus, le PCC a abandonné la formule selon laquelle les intervenantes et intervenants étaient les seules personnesressources des utilisatrices et utilisateurs du service; les bénévoles licenciés font dorénavant des heures de bureau sans rendez-vous, directement avec les personnes ciblées. Tandis que la structure du TDP était non hiérarchique, du moins en principe, le PCC a maintenant une directrice qui s'assure que ses politiques et pratiques sont conformes aux exigences du Barreau. Le PCC a été constitué en société sans but lucratif et est géré comme un partenariat communauté-campus. Finalement, n'étant pas une " clinique ${ }^{8}$ formelle et ayant un mandat très restreint (applicable uniquement aux avis d'infractions municipales et provinciales délivrés dans la région d'Ottawa), le PCC se différencie de la Clinique juridique communautaire de l'Université d'Ottawa, qui est très bien établie.

Les bénévoles du PCC ("les bénévoles accompagnateurs ") sont des avocates ou avocats ou des parajuristes dont la profession est réglementée et qui sont habilités à exercer le droit. Ils offrent des heures de consultation sans rendez-vous dans les locaux des partenaires communautaires, soit le Centre de santé communautaire du Centre-ville d'Ottawa, la Mission d'Ottawa, le Somerset West Community Health Centre et le Centre 454. Le PCC est en voie d'établir de nouveaux partenariats avec un refuge qui dessert la population itinérante francophone et un autre qui dessert la population itinérante d'origine autochtone. Cette façon de procéder permet aux bénévoles accompagnateurs de joindre plus facilement les personnes utilisatrices et, surtout, de les rencontrer dans un lieu qui leur est familier et facilement accessible.

Le PCC a maintenant un objectif explicitement pédagogique : former des étudiantes et étudiants en droit engagés et passionnés 
" Le PCC a

maintenant un

objectif explicitement

pédagogique : former

des étudiantes et

étudiants en droit

engagés et passionnés

par des causes sociales

et attirer de jeunes

avocates et avocats qui

aspirent à travailler

dans les domaines des

droits socioéconomiques

et de l'accès à la

justice. " par des causes sociales et attirer de jeunes avocates et avocats qui aspirent à travailler dans les domaines des droits socioéconomiques et de l'accès à la justice. Chaque bénévole accompagnateur est jumelé à deux étudiantes ou étudiants. Ces derniers observent le bénévole accompagnateur pendant les heures de bureau sans rendez-vous. Conformément au Code de déontologie du Barreau du Haut-Canada (règle 6-1.1) et aux règlements administratifs $\mathrm{n}^{\circ} 4$ et $\mathrm{n}^{\circ} 7.1$ de la Loi sur le Barreau, la directrice du PCC assure la supervision directe des étudiantes et étudiants en droit par l'entremise des bénévoles accompagnateurs, qui leur confient certaines tâches.

Les personnes utilisatrices ont rarement leurs avis d'infraction en main (puisqu'elles les ont jetés ou perdus). Ainsi, la première tâche de l'étudiante ou de l'étudiant est donc de se rendre au Bureau des infractions provinciales pour obtenir un rapport d'infraction du greffier. Ce document dresse un tableau de la situation juridique de la personne utilisatrice, notamment en indiquant si celle-ci a d'anciennes contraventions qui demeurent impayées ou si elle a reçu de nouveaux avis d'infraction, ou encore s'il existe une ordonnance qui exige sa présence devant le tribunal. Les autres responsabilités déléguées aux étudiantes et étudiants sont notamment : de réviser et de résumer le contenu du dossier du procureur, de recueillir tout élément de preuve supplémentaire, de mettre au point une position juridique au nom de la cliente ou du client, et d'informer la personne utilisatrice de son droit à une instance judiciaire bilingue. Les étudiantes et étudiants ne fournissent pas de conseils juridiques et ne se présentent pas comme avocates ou avocats, ou parajuristes. Cependant, ils peuvent, sous la supervision du bénévole accompagnateur, offrir de l'information juridique (par exemple démystifier le fonctionnement du tribunal, ses procédures, règlements et codes de conduite, vulgariser divers modes de résolution et donner des conseils sur la manière de se présenter devant le tribunal).

Lors d'une consultation, les bénévoles accompagnateurs offrent à la personne utilisatrice de l'information sur les diverses questions juridiques en jeu, l'aident à établir ses droits et lui présentent les diverses options qui s'offrent à elle, par exemple : contester 
son avis d'infraction, avec ou sans accompagnement du PCC, plaider coupable et/ou négocier l'allègement de l'amende. Si le problème juridique ne cadre pas avec le mandat du PCC (c'est-àdire la défense des avis d'infraction délivrés pour des motifs reliés à l'occupation de l'espace public ou autrement associés à une situation d'itinérance), mais se situe dans le domaine d'expertise de l'avocate ou de l'avocat ou du parajuriste accompagnateur, le bénévole peut lui offrir des services de représentation juridique bénévole. Le PCC compte parmi ses bénévoles accompagnateurs des expertes ou experts dans les domaines du droit pénal, des droits de la personne et du droit administratif. De surcroit, les bénévoles servent de source de référence à des organismes juridiques, telle la clinique juridique de l'Université d'Ottawa, qui est en mesure d'offrir des services plus spécialisés sur des questions liées au droit du logement ou au droit de l'immigration, par exemple.

Enfin, le PCC adopte une approche interdisciplinaire en ce qui a trait à la question de la judiciarisation de l'itinérance. Ses étudiantes et étudiants bénévoles sont non seulement des juristes en herbe, mais sont aussi inscrits dans des programmes de parajuristes, de criminologie et de service social. Ces étudiantes et étudiants, particulièrement celles et ceux qui proviennent des sciences sociales, contribuent à radicaliser le discours du PCC et à décentraliser l'aspect juridique des problèmes auxquels font face les personnes utilisatrices des services du PCC. Qui plus est, le PCC fonctionne autant que possible selon le modèle de réduction des méfaits et à partir d'une approche holistique. Par exemple, tous les étudiants et étudiantes reçoivent une formation interdisciplinaire en travail social, d'abord au sujet des approches qui tiennent compte des traumatismes (Trauma Informed Approaches), offerte par un de ses organismes partenaires, le Centre de santé communautaire du Centre-ville d'Ottawa. Les étudiantes et étudiants suivent également des formations sur l'emploi d'un langage approprié ainsi que sur les styles d'intervention qu'il convient d'adopter pour le travail avec des personnes atteintes de maladies mentales, par l'intermédiaire de l'organisme Connexion Ottawa9 . 
Du $1^{\text {er }}$ mai 2014 au 30 novembre 2015, le PCC a accueilli 47 personnes utilisatrices et est en voie de traiter 384 des avis d'infraction qui leur ont été délivrés en vertu de diverses lois provinciales et de divers règlements municipaux, et de règlements de la Commission de la capitale nationale (CCN).

\section{Profil des avis d'infraction délivrés aux personnes en situation d'itinérance à Ottawa du $1^{\mathrm{er}}$ mai 2014 au 30 novembre 2015}

"La raison d'être $d u$ PCC est d'offrir un meilleur accès à la justice aux personnes itinérantes d'Ottawa, en contestant la légitimité des lois et règlements provinciaux et municipaux relativement à l'occupation de l'espace public, au commerce sur le domaine public et à tout autre comportement associé à une situation d'itinérance."
La raison d'être du PCC est d'offrir un meilleur accès à la justice aux personnes itinérantes d'Ottawa, en contestant la légitimité des lois et règlements provinciaux et municipaux relativement à l'occupation de l'espace public, au commerce sur le domaine public et à tout autre comportement associé à une situation d'itinérance.

\section{Infractions provinciales}

En tout, 90 des avis d'infraction recueillis par les bénévoles du PCC du 1er mai 2014 au 30 novembre 2015 découlent de la mise en application de la Loi de 1999 sur la sécurité dans les rues : 52 concernent la sollicitation sur la chaussée de personnes dans un véhicule (al. 3(2)f)); 21 ciblent la sollicitation qualifiée d'" agressive " (par. 2(2)); 17 visent les individus qui sollicitent une personne en train de monter à bord d'un véhicule ou d'en descendre (al. 3(2)e)). Si nous pouvons nous fier aux données du SPO (2014) pour 2013, qui indiquent que le nombre total d'avis d'infraction délivrés en vertu de la LSR est de 2 296, il est clair que le PCC ne fait que voir la pointe de l'iceberg.

Une majorité des personnes utilisatrices du PCC ont été inculpées d'une infraction prévue par la Loi sur les permis d'alcool ("LPA »). En tout, le PCC a recueilli 116 avis d'infraction relatifs à la $L P A$, dont 59 pour ébriété publique (par. 31(4)) et 57 pour consommation d'alcool dans l'espace public (par. 31(2)).

D'autres avis d'infraction ont été délivrés en application de la Loi sur l'entrée sans autorisation ("LESA»): 49 pour entrée ou 
«Des 384 avis d'infraction recueillis par les bénévoles $d u$ PCC, 59 concernent la mise en application des règlements municipaux. " activité interdite dans un lieu public (al. 2(1)a)), 24 pour refus illégitime de quitter un lieu lorsque cela est ordonné (al. 2(1)b)) et 18 pour entrée dans un jardin, un champ ou une autre terre en culture, sans autorisation (sous-al. 32(1)a)i)). Les bénévoles du PCC ont également recueilli cinq procès-verbaux délivrés à des personnes utilisatrices en vertu du Code de la route, lequel exige qu'un vélo soit muni d'une sonnette et d'un réflecteur (par. 62(17)). Enfin, une seule contravention a été donnée en application de la Loi sur la protection de l'environnement, au titre de l'interdiction de déposer des matériaux susceptibles de devenir des détritus (art. 86).

\section{Infractions municipales}

Des 384 avis d'infraction recueillis par les bénévoles du PCC, 59 concernent la mise en application des règlements municipaux. Nous observons que 28 de ces avis se rapportent au défaut de présenter une preuve de paiement dans des véhicules du transporteur public OC Transpo sous le régime du Règlement sur le transport en commun (art. 11). Dix-sept avis d'infraction ont été délivrés en vertu du Règlement sur la circulation et le stationnement, lequel interdit de faire du vélo sur les trottoirs (art. 84). Finalement, 12 avis délivrés sous le régime du Règlement en matière de bruit se rattachent au fait de faire des bruits inhabituels (art. 2), et deux avis d'infraction ont étés délivrés en vertu du Règlement en matière de contrôle et de soin des animaux à une personne qui avait omis de faire enregistrer la possession d'un animal de compagnie (art. 7).

\section{Règlement sur les propriétés de la Commission de la capitale nationale}

Les bénévoles du PCC ont traité 22 avis d'infraction touchant le Règlement sur les propriétés de la Commission de la capitale nationale et la circulation sur ces dernières. Cinq avis d'infraction ont trait à la possession d'alcool en violation des lois provinciales, et trois avis se rapportent à l'interdiction de mettre en vente des objets ou des marchandises sans avoir préalablement obtenu la permission de la Commission (art. 32). Les personnes utilisatrices du PCC 
se sont également vu délivrer quatre avis pour avoir campé ou pique-niqué ou pour avoir érigé une tente sur les terrains de la Commission (art. 38). Les avis d'infraction visant à punir l'adoption d'une conduite choquante (art.9), la possession de biens provenant du domaine de la Commission (art. 26), ainsi que le fait d'alimenter un feu (art. 30), ne représentent qu'un très petit nombre, soit un avis par catégorie pour un total de trois.

L'article 32 du Règlement sur les propriétés de la Commission de la capitale nationale et la circulation sur ces dernières pose des difficultés particulières pour les personnes en situation d'itinérance en ce qu'il punit la sollicitation tout court. Cette disposition se lit comme suit :

32. Nul ne doit, à moins d'en avoir reçu la permission écrite de la Commission, vendre, mettre en vente ou étaler pour la vente, des boissons, des objets, ou des marchandises, ni afficher ou exposer des enseignes, placards, drapeaux ou réclames, ni solliciter des abonnements ou versements sur ou dans un terrain de la Commission.

(Le soulignement est de nous.)

Le fardeau de la preuve est moins lourd pour les poursuivants qui doivent établir qu'une personne sollicitait des versements sur ou dans un terrain de la Commission de la capitale nationale (solicit contributions on or in any property) et non qu'elle sollicitait d'une façon prohibée, comme la sollicitation qualifiée d'" agressive " sous le régime de la $L S R$.

Jusqu'à maintenant, le PCC n'a recueilli que sept avis d'infraction relatifs à l'article 32 , mais l'auteure de la présente recherche prévoit que le PCC en verra beaucoup plus si le mouvement pour abolir la LSR prend de l'ampleur. Rappelons qu'en 2007 les tribunaux ont conclu à la constitutionnalité de plusieurs dispositions de la LSR (R. c. Banks). Depuis, un grand nombre de groupes et d'intervenantes et intervenants, dont la Coalition to Repeal the Safe Streets Act, militent en faveur de son abrogation (Wagner, 2015). 
Qui plus est, Cheri DiNovo, députée néo-démocrate du parlement provincial, a déposé le projet de loi 94 à l'Assemblée législative de l'Ontario le 29 avril 2015. Tenant son nom de l'ancien député provincial Peter Kormos, le projet de loi 94 visait à abroger la très polémique LSR. À l'étape de la première lecture du processus législatif, qui correspond au dépôt du projet de loi devant l'Assemblée législative, le projet de loi a été adopté à l'unanimité, laissant présager une abrogation imminente de la Loi de 1999. Toutefois, il est fréquent que les propositions parlementaires franchissent l'étape de la première lecture. Tant que le projet de loi n'aura pas été entériné, la LSR demeurera en vigueur. Le gouvernement de la première ministre Kathleen Wynn ne s'est toujours pas prononcé sur la motion parlementaire de la députée DiNovo.

\section{Obstacles systémiques et sociaux à la contestation des contraventions}

"Les personnes en situation d'itinérance

à Ottawa qui veulent contester leurs contraventions sans accompagnement se heurtent à des obstacles systémiques et sociaux qui limitent sérieusement leur capacité d'exercer leurs droits. »
Les personnes en situation d'itinérance à Ottawa qui veulent contester leurs contraventions sans accompagnement se heurtent à des obstacles systémiques et sociaux qui limitent sérieusement leur capacité d'exercer leurs droits. Elles font face à un système de justice particulièrement complexe, au sein duquel il est difficile de naviguer sans l'aide de services juridiques. D'abord, les services d'Aide juridique Ontario (un organisme autonome sans but lucratif financé par l'État, qui offre des services juridiques aux personnes à faible revenu) et les avocates et avocats de service (duty counsel) (qui offrent sur-le-champ une aide juridique aux personnes à faible revenu qui se présentent au tribunal sans avocat) sont rarement disponibles en matière d'infractions provinciales quand il n'y a pas de risque réel d'emprisonnement (Bouclin, 2014). Qui plus est, nombreuses sont les personnes itinérantes qui ont déjà été victimes d'intimidation et de harcèlement de la part des policiers. Par conséquent, l'idée de témoigner en cour contre un policier peut être une tâche extrêmement ardue, et une personne peut préférer laisser tomber la contestation par crainte de représailles (Bouclin, 2014). Par surcroît, se présenter 
"À ce jour, parmi

les 47 personnes représentées par le PCC, lorsqu'il y a eu un procès, seulement une personne a dî payer l'amende, laquelle a été réduite de plusieurs centaines de dollars. " en cour peut s'avérer un défi de taille pour celles et ceux qui souffrent de mobilité réduite ou qui n'ont pas les moyens de payer les frais de transport pour se rendre personnellement au palais de justice, puisqu'ils se retrouvent à plusieurs kilomètres des quartiers du centre-ville, l'endroit où la plupart des contraventions sont données (Bouclin, 2014). Finalement, certaines personnes itinérantes ne peuvent adhérer au code vestimentaire de la cour, ou n'ont pas les connaissances ni la confiance nécessaires pour naviguer au sein du système de justice (Bouclin, 2014).

Le PCC tente de combler un besoin juridique de trois façons. D'abord, il fournit aux personnes en situation d'itinérance une représentation juridique devant les tribunaux de l'Ontario au regard des infractions reliées à la pauvreté. Le PCC obtient des résultats impressionnants, particulièrement en ce qui concerne le retrait des accusations contre les personnes que ses bénévoles accompagnent ou représentent. Dans les rares cas où les personnes utilisatrices sont reconnues coupables, le PCC a réussi à obtenir une suspension de leur peine. À ce jour, parmi les 47 personnes représentées par le PCC, lorsqu'il y a eu un procès, seulement une personne a dû payer l'amende, laquelle a été réduite de plusieurs centaines de dollars. Ensuite, si les personnes utilisatrices désirent se représenter elles-mêmes, le PCC offre des services tels que l'accompagnement au tribunal, la prestation de conseils pour leur permettre d'élaborer leurs arguments juridiques et la communication de renseignements sur la procédure judiciaire. Enfin, si la personne itinérante fait face à un problème qui ne cadre pas avec le mandat du PCC, celui-ci l'aiguillera vers des avocates ou avocats ou des centres communautaires susceptibles de l'aider.

\section{Défis, perspectives d'avenir et conclusions}

Le principal défi pour le PCC est la précarité. Le PCC n'est pas une clinique juridique formelle. Instituées dans les années 1970, les cliniques juridiques ontariennes sont des organismes sans but lucratif qui parviennent à financer leurs activités grâce aux subventions annuelles qu'elles reçoivent d'Aide juridique Ontario $(\mathrm{AJO})^{10}$. Pour sa part, le PCC ne réunit pas une équipe permanente rémunérée. Il dépend entièrement des bénévoles qui souhaitent 
jouer un rôle en tant qu'agents de changements sociaux, que ce soient des avocates et avocats, des parajuristes titulaires d'un permis d'exercice, des étudiantes et étudiants, ou des employées et employés de nos partenaires communautaires qui font des heures supplémentaires pour assurer le bon fonctionnement du programme.

Le fait de pouvoir compter sur l'appui et le dévouement de bénévoles constitue une valeur ajoutée intéressante pour une organisation. Ce trait particulier représente une force en soi pour le PCC, car celui-ci peut mener ses activités à terme sans pour autant avoir à se soucier de sa santé financière. Toutefois, plusieurs éléments font qu'un tel modèle est fragile et que la survie de l'organisation peut être mise en péril à tout moment. La grande majorité des bénévoles étant des étudiantes et étudiants, la participation des membres du PCC risque d'être éphémère. Après leurs études, certains décideront de continuer à participer aux activités du PCC, alors que d'autres y renonceront. Cette structure pose aussi des défis organisationnels en ce qui concerne la mémoire institutionnelle. Par ailleurs, les services du PCC sont de plus en plus recherchés, et ce dernier compte donc nouer trois nouveaux partenariats d'ici à la fin de 2016, soit avec un organisme desservant la population autochtone, avec un refuge pour femmes et avec un organisme offrant des services et des programmes en français. En outre, certains juges de paix, sans doute bien intentionnés, adressent au PCC des personnes qui se représentent elles-mêmes après s'être vu remettre un procèsverbal d'infraction. Mais, étant donné que les juges de paix ne connaissent pas la structure du PCC, soit des heures de bureau fixes qui permettent aux bénévoles d'obtenir un mandat (retainer), le PCC est rarement en mesure d'aider ces personnes.

Un second défi relève justement du modèle d'heures de bureau fixes, même sans rendez-vous, qui ne répond pas toujours aux besoins des personnes en situation d'itinérance. Il faudrait à tout prix que le PCC évolue à cet égard, ce qu'il a tenté de faire l'été dernier. Le 24 juin 2015, le PCC a participé à la campagne "Soutenez. Ne punissez pas. " (Support. Don't Punish.), une campagne mondiale visant à promouvoir l'adoption de meilleures 
politiques en matière d'usage de drogues axées sur la santé publique, les droits de la personne et la réduction des méfaits. Le PCC a collaboré à l'organisation ${ }^{11}$ d'une journée d'action et de mobilisation visant à contester les contraventions données aux personnes opprimées et ciblées, notamment les personnes itinérantes, par les actrices et acteurs du système de justice. Au cours de cette journée, les personnes en situation d'itinérance ont pu obtenir de l'information juridique gratuite ainsi que des renseignements sur la manière de se faire accompagner ou représenter en cour relativement aux avis d'infraction délivrés en vertu des lois ciblant l'occupation des espaces publics. L'événement a permis aux clientes et clients éventuels qui ne peuvent se présenter pendant les heures d'ouverture sans rendez-vous de la clinique mobile de bénéficier des conseils d'une avocate ou d'un avocat, et des étudiantes et étudiants du programme. Cette journée a été aussi, grâce à la couverture médiatique, une occasion de sensibiliser le public au profilage social des personnes en situation d'itinérance.

Malgré cette réussite, la mobilisation a aussi mis en relief la difficulté d'élaborer des réponses ciblées pour combler les besoins des personnes utilisatrices du service, tout en satisfaisant aux exigences du Barreau, c'est-à-dire d'offrir des services de représentation juridique, par l'intermédiaire des titulaires d'un permis d'exercice du droit, à une population marginalisée, stigmatisée et souvent transitoire, et d'assurer un suivi. Sur les 22 personnes avec lesquelles le PCC a pu faire du travail sur place, presque la moitié d'entre elles n'ont pu se rendre aux locaux du PCC pendant les heures de bureau sans rendez-vous pour signer la convention de mandat qui permettrait aux bénévoles accompagnateurs de s'occuper de leurs dossiers. Un défi connexe est la difficulté d'assurer une communication efficace avec la clientèle du PCC. Pour être en mesure de communiquer avec les personnes utilisatrices afin de les tenir au courant de l'état de leur dossier, le PCC dépend grandement des employés de ses partenaires communautaires, qui sont déjà débordés.

Un dernier défi se pose. Le PCC semble adopter une optique d'accès à la justice orthodoxe et à court terme, qui donne un accès 
"Ce faisant, il y a un risque qu'on légitime ou normalise les pratiques de profilage social en créant une infrastructure qui répond aux besoins juridiques des personnes itinérantes au lieu de contester les lois et les politiques qui posent problème."

"Chose certaine, par son caractère unique en soi, le PCC doit à tout prix éviter de sombrer dans le formalisme pour ainsi garder toute sa pertinence et son utilité pour les personnes utilisatrices de ses services, lesquels demeurent essentiels pour la communauté itinérante. » aux avocates et avocats, et aux parajuristes titulaires d'un permis d'exercice qui sont aptes à représenter des personnes en situation d'itinérance devant les tribunaux. Ce faisant, il y a un risque qu'on légitime ou normalise les pratiques de profilage social en créant une infrastructure qui répond aux besoins juridiques des personnes itinérantes au lieu de contester les lois et les politiques qui posent problème. Il s'agit là d'un enjeu qui doit demeurer au premier plan, si le PCC veut maintenir sa vision et sa mission originales, qui consistent à dénoncer les injustices à l'endroit des personnes en situation d'itinérance. Dans cette optique, les bénévoles du PCC offrent aux poursuivantes et poursuivants, et aux juges de paix une formation au sujet du profilage social et travaillent avec leurs partenaires communautaires, lesquels se mobilisent pour exercer des pressions sur les gouvernements provinciaux et les administrations municipales afin qu'ils investissent davantage dans des programmes sociaux plutôt que de s'en remettre à la judiciarisation. Le PCC va bientôt lancer, en collaboration avec ses partenaires communautaires, un projet dont l'objet est de surveiller et de documenter la brutalité policière et de manifester contre celle-ci. Il suit également avec grand intérêt le Programme d'accompagnement justice pour les personnes itinérantes à la Cour municipale de la Ville de Montréal (PAJIC) afin d'évaluer dans quelle mesure Ottawa pourrait adopter un modèle semblable. Le PCC en est encore au stade du développement, de sorte qu'il est important de laisser la chance au coureur. Chose certaine, par son caractère unique en soi, le PCC doit à tout prix éviter de sombrer dans le formalisme pour ainsi garder toute sa pertinence et son utilité pour les personnes utilisatrices de ses services, lesquels demeurent essentiels pour la communauté itinérante.

En juin 2015, le Service de police d'Ottawa a mené deux campagnes de "tolérance zéro ", d'une durée de cinq jours chacune, visant officiellement à réprimer l'activité criminelle et les infractions aux lois provinciales (SPO, 2015b). Ces campagnes ont été, dans les faits, une stratégie d'identification, d'interception et de dispersion des personnes en situation d'itinérance. Dans la première campagne, 338 " avis d'infractions " provinciales et municipales ont été délivrés (SPO, 2015b). Ils portent sur les 
infractions provinciales visées par la $L S R$ ainsi que par la Loi sur les permis d'alcool (LPA) et la Loi sur l'entrée sans autorisation (LESA) et sur plusieurs infractions aux règlements municipaux (SPO, 2015b) pour des motifs liés à l'occupation de l'espace public, à savoir : avoir en sa possession de l'alcool (bouteille débouchée) dans un lieu public, être un piéton indiscipliné (" traverser illégalement la chaussée »), ne pas avoir de médaille d'identification pour un animal de compagnie, utiliser des moyens de transport public sans preuve d'achat, ne pas porter un casque protecteur en vélo, être coupable d'intrusion (se trouver sans autorisation sur une propriété publique ou privée) et faire des bruits perturbateurs. Lors de la deuxième campagne, 116 avis d'infractions provinciales ont été délivrés, portant notamment sur les infractions aux mêmes lois que celles visées lors de la première campagne (SPO, 2015a). Ces contraventions sont punissables d'une amende pouvant atteindre $1000 \$$, en sus des frais administratifs et d'une suramende compensatoire. Ce chiffre, soit 454 avis d'infraction en cinq jours, n'est qu'une fraction des contraventions données aux personnes en situation d'itinérance à Ottawa. Par ailleurs, d'avril 2014 à mars 2015, dans la ville d'Ottawa, 1085 avis d'infraction ont été délivrés en vertu de la $L S R$, et on peut raisonnablement présumer qu'ils ont tous été remis à des personnes itinérantes (Cour de justice de l'Ontario, 2016, p. 36). Pendant la même période, 3940 avis d'infraction ont été délivrés sous le régime de la $L P A$, dont bon nombre aux personnes en situation d'itinérance (Cour de justice de l'Ontario, 2016, p. 36).

Le PCC offre des services d'intervention juridique et communautaire importants à un groupe marginalisé et stigmatisé. Ces services sont gratuits et offrent un accès direct à la justice aux personnes en situation d'itinérance. La nécessité pour le PCC d'offrir ces services croît au fur et à mesure que les partenaires communautaires ont connaissance du travail accompli par le PCC et constatent son succès relatif. L'élargissement de sa vocation prochaine pour lui permettre d'œuvrer auprès des personnes francophones et des personnes d'origine autochtone en situation d'itinérance de la ville d'Ottawa non seulement favorisera l'accès à la justice d'une communauté mal desservie, mais également aidera 
à documenter le profilage social à Ottawa grâce à la collecte de données sur le nombre de contraventions dressées aux utilisateurs des services du PCC.

\section{Notes}

1 Cette recherche a grandement bénéficié de la contribution de nombreux étudiants, étudiantes et stagiaires du PCC, dont Patrick Lavoie, Marie-Andrée Denis-Boileau et Isabelle Hardy. Merci surtout aux personnes utilisatrices des services du PCC. J'aimerais exprimer ma gratitude aux membres du comité de rédaction et aux évaluatrices et évaluateurs pour leurs commentaires judicieux et constructifs. Toutes les erreurs ou omissions sont les miennes.

2 La définition d'" agent des infractions provinciales » est vaste et permet d'inclure les agents de police, les agents d'exécution des règlements municipaux, etc. (par. 1(1) de la LIP).

3 La Loi sur les infractions provinciales, LRO 1990, c P.33 («LIP») régit la procédure à suivre concernant les infractions prévues par la $L S R$. L'objet de la LIP est de remplacer la procédure de déclaration de culpabilité par procédure sommaire "par une procédure qui reflète la distinction existant entre les infractions provinciales et les infractions criminelles ».

4 Document non publié en possession de l'auteure.

5 Le PCC voit régulièrement des personnes débitrices de plus de $1000 \$$ en amendes impayées (documents non publiés en possession de l'auteure).

6 La Cour de justice de l'Ontario et la Cour supérieure de justice (qui entend les causes civiles et les causes portant sur les infractions graves prévues au Code criminel du Canada) sont les deux tribunaux de première instance de l'Ontario. La Cour de justice de l'Ontario est composée de juges et de juges de paix. Les juges de paix ont compétence pour entendre les causes qui s'inscrivent dans le mandat du TDP, soit celles relatives aux infractions provinciales et municipales. Les juges de la Cour ont compétence pour instruire les affaires touchant le droit de la famille ainsi que la grande majorité des accusations criminelles déposées en Ontario : http://www. ontariocourts.ca/ocj/fr/infractions-provinciales/apercu/ (2016).

7 La Loi prévoit dès 2006 que le tribunal peut exclure " quiconque, à l'exception d'une personne pourvue d'un permis délivré en vertu de la Loi sur le Barreau » et lui interdire " de comparaitre comme représentant, s'il conclut que cette personne n'a pas la compétence voulue pour représenter ou conseiller la personne au nom de laquelle elle comparait, ou ne comprend ni n'observe les devoirs et les responsabilités d'un représentant " (par. 51(6), LIP).

8 Nous examinerons plus loin pourquoi le PCC ne répond pas aux critères des cliniques juridiques communautaires.

9 Cet organisme a comme objectif d'améliorer l'accès à la justice pour les personnes qui ne parlent ni l'anglais ni le français et «qui doivent affronter des défis de communication en raison d'un handicap ou d'une déficience sensorielle»(Voir : http://connexionottawa.com/propos-de-nous).

10 Ayant pris forme grâce au Programme des cliniques juridiques communautaires, elles jouent un rôle important tant pour les communautés anglophones que francophones de la province. À ce jour, l'Association des cliniques juridiques communautaires de l'Ontario (ACJCO) soutient que le nombre de CJC en Ontario est de 77 (ACJCO, 2014). 
11 La journée «Ticket Defence Action - Stand Against Tickets in Minto Park » a été organisée en partenariat avec le Centre de santé communautaire du Centre-ville d'Ottawa, le Somerset West Community Health Centre et le Comité sida d'Ottawa.

\section{Bibliographie}

ALLIANCE POUR METTRE UNTERME À L'ITINÉRANCE À OTTAWA (ATEHO) (2009). Bulletin 2009, réf. du 30 janvier 2015, http://endhomelessnessottawa.ca/wpcontent/uploads/2014/04/

BulletinpourmettreuntermealitineranceaOttawaJanDec2009.pdf

ALLIANCE POUR METTRE UN TERME À L'ITINÉRANCE À OTTAWA (ATEHO) (2014). Bulletin 2013, réf. du 5 avril 2016, http://endhomelessnessottawa.ca/wp-content/ uploads/2014/04/2013-FR-alliance-to-end-homelessness-fr-digital.pdf

ALLIANCE POUR METTRE UNTERME À L'ITINÉRANCE À OTTAWA (ATEHO) (2015). Bulletin 2014, Progress Report on Ending Homelessness in Ottawa, réf. du 30 janvier 2015, http:// endhomelessnessottawa.ca/2014-progress-report/

AMNISTIE INTERNATIONALE (2009). Assez de vies volées! Discrimination et violence contre les femmes autochtones au Canada : Une réaction d'ensemble est nécessaire, réf. du 13 mai 2016, http://amnistie.ca/sites/default/files/upload/documents/publications/ amr200122009fraassezdeviesvolees.pdf

ASSEMBLÉE DES PREMIÈRES NATIONS DU QUÉBEC ET DU LABRADOR (APNQL) ET LA COMMISSION DE LA SANTÉ ET DES SERVICES SOCIAUX DES PREMIÈRES NATIONS DU QUÉBEC ET DU LABRADOR (CSSSPNQL) (2008). Le phénomène de l'itinérance chez les Premières Nations au Québec, réf. du 8 octobre 2015, http://www.pvsq.org/articles/Memoire-itinerance.pdf

ASSOCIATION DES CLINIQUES JURIDIQUES COMMUNAUTAIRES DE L'ONTARIO (ACJCO) (2014). About Community Legal Clinics - Overview, réf. du 30 janvier 2015, http://www.aclco.org/about_Clinics_overview.html

ASSOCIATION DES ÉTUDIANT(E)S INFIRMIER(ÈRE)S DU CANADA (AEIC) (2014). Santé et itinérance, réf. du 30 mars 2016,

http://www.aeic.ca/francais/aboutus/policies-and-position-statements/position-statements/ health-and-homelessness

AUBRY, Tim, et collab. (2008). Un toit à nouveau, [rapport de recherche], Université d'Ottawa, Carleton University, Alliance pour mettre un terme à l'itinérance à Ottawa,Ville d'Ottawa, 14 p.

BELANGER, Yale D., Olu AWOSOGA et Gabrielle WEASEL HEAD (2013). "Homelessness, Urban Aboriginal People, and the Need for a National Enumeration ", Aboriginal Policy Studies, Vol. 2, No 2, p. 4-33.

BELLOT, Céline, et collab. (2007). La judiciarisation des populations itinérantes en Ontario, [rapport de recherche], Secrétariat national des sans-abri, $58 \mathrm{p}$.

BELLOT, Céline, et Marie-Ève SYLVESTRE (2012). La judiciarisation des populations itinérantes à Montréal, [rapport de recherche], Montréal, Université de Montréal, et Ottawa, Université d'Ottawa, 37 p. 
BERNIER, Dominique, et collab. (2011). La judiciarisation des personnes en situation d'itinérance à Québec: point de vue des acteurs socio-judiciaires et analyse du phénomène, [rapport de recherche Homelesshub], Toronto, The Canadian Homelessness Research Network Press, p. 1-44.

BLOMLEY, Nicholas (2010). «The Right to Pass Freely: Circulation, Begging, and The Bounded Self ", Social \& Legal Studies, Vol. 19, No 3, p. 331-350.

BOUCLIN, Suzanne (2014). «Regulated Out of Existence:A Case Study of Ottawa's Ticket Defence Program ", Journal of Law and Equality, Vol. 11, p. 35-83.

BOUCLIN, Suzanne (2015). "Identifying Pathways to and Experiences of Street Involvement through Case law ", Dalhousie Law Journal, Vol. 38, Nº 2, p. 345-83.

BOUCLIN, Suzanne, et Joëlle P. SALA (2013). «Une analyse de la réglementation des personnes itinérantes selon une perspective pothienne de la discrimination ", Revue de la femme et du droit, Vol. 25, $\mathrm{N}^{\mathbf{0}} 1$, p. 1-30.

BOYLE, Theresa (1999). «Tories to take swipe at squeegee kids; Proposed Law Targets Aggressive Panhandlers", The Toronto Star, p.A7.

BROWN, Joyce, et Diana CAPPONI (1993). "Housing Homeless Women in Toronto ", dans Hemalata C. Dandekar (dir.), Shelter, Women and Development: First and Third World Perspectives, Ann Arbor, MI, George Wahr Publishing, p. 152-156.

CAMPBELL, Christine, et Paul EID (2009). La judiciarisation de personnes itinérantes à Montréal : un profilage social, réf. du 20 mai 2015, http://www.cdpdj.qc.ca/Publications/itinerance_avis.pdf

CENTRE 454 (2013). Centre 454, réf. du 29 septembre 2015, http://www.centre454.ca/index. php/en/

CENTRE DE SANTÉ COMMUNAUTAIRE DU CENTRE-VILLE D'OTTAWA (2015). À propos du CSC, réf. du 6 avril 2016, http://www.centretownchc.org/fr/le-centre.aspx

CHESNAY, Catherine T., Céline BELLOT et Marie-Ève SYLVESTRE (2013). «Taming Disorderly People One Ticket at a Time: The Penalization of Homelessness in Ontario and British Columbia ", Canadian Journal of Criminology and Criminal Justice, Vol. 55, No 2, p. 161-185.

COLLINS, Damian, et Nicholas BLOMLEY (2003). "Private Needs and Public Space: Politics, Poverty, and Anti-Panhandling By-Laws in Canadian Cities ", dans Commission du droit du Canada, New Perspectives on the Public-Private Divide,Vancouver, UBCPress, p. 40-67.

COMITÉ DES SANS-ABRI (1987). Vers une politique municipale pour les sans-abri, [rapport de recherche], Montréal, Conseil municipal de la Ville de Montréal, 64 p.

COMMISSION DE LA CAPITALE NATIONALE (CCN-NCC). La région de la capitale du Canada, réf. du 7 octobre 2015, http://www.ccn-ncc.gc.ca

COMMISSION DE LA CAPITALE NATIONALE (CCN-NCC). Plan de secteur du cœur de la capitale du Canada, réf. du 2 avril 2016, http://www.ccn-ncc.gc.ca/planifier/plans-directeurs/ plan-de-secteur-du-coeur-de-la-capitale-du-canada

COMMISSION DE LA CAPITALE NATIONALE (CCN-NCC). Terrains urbains de la capitale, réf. du 7 octobre 2015, http://www.ccn-ncc.gc.ca/planifier/plans-directeurs/terrains-urbainsde-la-capitale

COMMISSION DES DROITS DE LA PERSONNE ET DES DROITS DE LA JEUNESSE (CDPDJ) (2009). La judiciarisation des personnes itinérantes à Montréal : un profilage social, réf. du 7 octobre 2015, http://www.cdpdj.qc.ca/publications/Fiche_1_profilage_definition.pdf 
COUR DE JUSTICE DE L'ONTARIO, Aperçu, réf. du $1^{\text {er }}$ avril 2016, http://www.ontariocourts.ca/ocj/fr/infractions-provinciales/apercu/

COUR DE JUSTICE DE L'ONTARIO. À propos de la Cour, réf. du 11 avril 2016, http://www.ontariocourts.ca/ocj/fr/ocj/a-propos-de-la-cour/

CROSS, Jessica S. (2014). «Panhandling tickets saddle Toronto's homeless with debt:York professor », Metro News, réf. du 20 mai 2014, http://www.metronews.ca/news/toronto/2014/12/15/ panhandling-tickets-saddle-torontos-homeless-with-debt-york-professor.html

CZAPSKA, Asia, Annabel WEBB et Nura TAEFI (2008). More than Bricks \& Mortar:A Rights-Based Strategy to Prevent Girl Homelessness in Canada, Vancouver, Justice for Girls, 65 p.

ECHENBER G, Havi, et Hilary JENSEN (2009). «Échec du mariage et relations violentes », dans la Bibliothèque du Parlement, Facteurs de risque de l'itinérance, Ottawa, Division des affaires sociales, Service d'information et de recherches parlementaires, p. 1-10.

ESMONDE, Jackie (2002). «Criminalizing Poverty: The Criminal Law Power and the Safe Streets Act ", Journal of Law and Social Policy,Vol. 17, p. 63-86.

FARRELL, Susan.J.,Tim AUBRY et Elke REISSING (2001). Street needs assessment:An investigation of the characteristics and service needs of persons who are homeless and not currently using emergency shelter in Ottawa, [rapport de recherche], Ottawa, Centre for Community Research.

FÉDÉRATION EUROPÉENNE DES ASSOCIATIONS NATIONALESTRAVAILLANT AVEC LES SANS-ABRI (FEANTSA) (2015). Mean Streets : un rapport sur la criminalisation des sans-abri en Europe, [rapport de recherche], Bruxelles, Belgique, 16 p.

GAETZ, Stephen, et collab. (2013). État de l'itinérance au Canada 2013, [rapport de recherche Homelesshub], Toronto, The Canadian Homelessness Research Network Press, $52 \mathrm{p}$.

GÉLINEAU, Lucie, et collab. (2006). "Le droit à sa place ", Recherches féministes, Vol. 19, № 2, p. 125-141.

GERMAIN, Marie-France, Rosalinda COSTA et Karen KELLY-SCOTT (2009). Profil de la population autochtone de 2006 pour Ottawa, [rapport de recherche], Ottawa, ministre de l'Industrie, Statistique Canada, $22 \mathrm{p}$.

GLOWINSKI, Kent (2009). «Don't Get Enough Credit? The Need for an Impartial Consumer Credit Report Appeal Tribunal in Ontario ", Journal of Law and Social Policy, Vol. 22, Nº 1, p. 5-25.

GOFFMAN, Alice (2014). On the Run, Fugitive Life in an American City, Fieldwork Encounters and Discoveries, $288 \mathrm{p}$.

HERMER, Joe, et Janet E. Mosher (2002). Disorderly People: Law and the Politics of Exclusion in Ontario, Halifax, Fernwood, 121 p.

KAUPPI, Carol, et collab. (2012). « Niveaux et raisons du sans-abrisme chez les Francophones dans le nord de l'Ontario ", Reflets : revue d'intervention sociale et communautaire, Vol. 18, No 1, p. 91-118.

KIRKUP, Kristy (2013). "Half of Homeless Ottawa youth identify as LGBTQ », CBCnews Ottawa, réf. du 2 avril 2016, http://www.cbc.ca/news/canada/ottawa/half-of-homeless-ottawa-youthidentify-as-lgbtq-1.1699604

KLOS, Nancy (1997). "Aboriginal Peoples and Homelessness: Interviews with Service Providers ", Canadian Journal of Urban Research, Vol. 6, N 1, p. 40-52. 
KNOTSCH, Cathleen, et Dianne KINNON (2011). If Not Now...When? Addressing the Ongoing Inuit Housing Crisis in Canada, [rapport], Ottawa, Canada, National Aboriginal Health Organization, $74 \mathrm{p}$.

LABER GE, Danielle, Mario POIRIER et René CHAREST (1998). "Un étranger dans la cité : la présence de l'itinérant et la représentation de l'itinérance ", Nouvelles pratiques sociales, Vol. $11, N^{\mathbf{o}} 1$, p. $19-24$.

LAROUSSE, subverbo (2015). «judiciarisation », réf. du 20 avril 2015, http://www.larousse.com/en/dictionnaires/francais/judiciarisation/45098

LENON, Suzanne (2000). "Living on the Edge: Women, Poverty and Homelessness in Canada ", Canadian Woman Studies, Vol. 20, No 3, p. 123-126.

MAISON D'AMITIÉ (2013). Mandat et missions de la Maison d'amitié, réf. du 20 mars 2016, http://www.maisondamitie.ca/index.cfm?Voir=sections\&Id=9166\&M=2809\&Repertoi re_No=2137988399

MENZIES, Peter M. (2005). Orphaned within our families: Intergenerational trauma and homeless among aboriginal men, [thèse de doctorat], Toronto, Canada, Université de Toronto, $2406 \mathrm{p}$.

MINISTÈRE DU PROCUREUR GÉNÉRAL (2015). Contraventions et amendes, réf. du 5 avril 2016, https://www.attorneygeneral.jus.gov.on.ca/french/justice-ont/tickets_and_fines.php

MINISTÈRE DU PROCUREUR GÉNÉRAL (2001). Rapport annuel 2001 du vérificateur provincial de l'Ontario :Aide juridique Ontario, Bureau du vérificateur général de l'Ontario, réf. du 20 février 2016, http://www.auditor.on.ca/fr/rapports_fr/fr01/302fr01.pdf

MOLGAT, Marc (1995). «Des jeunes à l'écart du social? Histoires des jeunes francophones sans abri à Ottawa-Carleton ", Reflets : revue d'intervention sociale et communautaire, Vol. 1, No 1, p. 141-163.

MOLNAR, Beth E., et collab. (1998). «Suicidal Behavior and Sexual/Physical Abuse among Street Youth ", Child Abuse and Neglect, Vol. 22, № 3, p. 213-222.

MOON, Richard (2002). « Keeping the Streets Safe from Free Expression », dans Joe Hermer et Janet E. Mosher, Disorderly People: Law and the Politics of Exclusion in Ontario, Halifax, Fernwood, p. 65-78.

NATIONAL ABORIGINAL HEALTH ORGANIZATION (2006). NAHO, réf. du 2 avril 2016, http://www.naho.ca

NOVAC, Sylvia (2006). Violence familiale et itinérance : Analyse documentaire, Centre national d'information sur la violence dans la famille, [rapport de recherche], Ottawa :Agence de la santé publique du Canada, $59 \mathrm{p}$.

NOVAC, Sylvia, et collab. (2002). Où se tourner? La situation des jeunes femmes sans abri au Canada, [rapport de recherche], Condition féminine Canada, $173 \mathrm{p}$.

O'GRADY, Bill, Stephen GAETZ et Kristy BUCCIERI (2011). Can I See your ID? The Policing of Youth Homelessness in Toronto, [rapport de recherche], Toronto, Justice for Children and Youth, and The Canadian Homelessness Research Network Press, p. 1-93.

OSUJI, Joseph C., et Sandra P. HIRST (2013). «Understanding the Journey through Homelessness: A Hermeneutic Study of Women Without Children ", Canadian Journal of Community Mental Health, Vol. 32, $\mathrm{N}^{\mathrm{o}}$ 2, p. 27-42.

OTTAWA (2014). Rapport final — "Cercles d'écoute », réf. du 11 avril 2016, http://ottawa.ca/fr/hotelde-ville/votre-administration-municipale/comite-detude-sur-les-questions-autochtones/rapport 
OTTAWA (2014). Sans-abrisme, réf. du 20 mars 2016, http://ottawa.ca/fr/residents/services-sociaux/ logements/sans-abrisme

OTTAWA (2015). Addressing Homelessness, réf. du 4 avril 2016, http://ottawa.ca/en/residents/socialservices/housing/addressing-homelessness

OTTAWA MISSION (2016). Ottawa Mission, réf. du 6 avril 2016, http://ottawamission.com/

PICHON, Pascale (2009). SDF, sans-abri, itinérant : Oser la comparaison, Collection Atelier de recherches sociologiques (ARS), Presses universitaires de Louvain, $194 \mathrm{p}$.

POUPART, Jean (dir.) (2004). Au-delà du système pénal, l'intégration sociale et professionnelle des groupes judiciarisés et marginalisés, Québec, Presses de l'Université du Québec, 294 p.

POUPEAU, Franck, et Sylvie TISSOT (2005). "La spatialisation des problèmes sociaux ", Actes de la recherche en sciences sociales, $\mathrm{N}^{\circ} 159$, septembre 2005, p. 5-9.

PROGRAMME D'ACCOMPAGNEMENT JUSTICE POUR LES PERSONNES ITINÉRANTES À LA COUR (PAJIC) (2011). PAJIC, réf. du 20 mars 2015, http://www.justice.gouv.qc.ca/francais/themes/jsm/pdf/prog_procureur.pdf

REGROUPEMENT DES CENTRES D'AMITIÉ AUTOCHTONES DU QUÉBEC (2008). Mémoire sur le phénomène de l'itinérance chez les Autochtones en milieu urbain du Québec, réf. du 18 mars 2016, http://www.rcaaq.info/images/stories/docs/memoire_itinerance.pdf

RÉSEAU CANADIEN DE RECHERCHE SUR L'ITINÉRANCE (2013). La définition canadienne de l'itinérance, [rapport], Canadian Homelessness Research Network, 5 p.

RÉSEAU CANADIEN POUR LA SANTÉ DES FEMMES (2003). "L'ampleur du problème de l'itinérance chez les femmes est sous-estimée, révèle une étude ", réf. du 2 mai 2016, http://www.cwhn.ca/fr/node/39986

ROY, Shirley (2009). «Histoire politique de la question itinérante au Québec », dans Pascale PICHON (dir.), SDF, sans-abri, itinérant : oser la comparaison, Collection Atelier de recherches sociologiques (ARS), Presses universitaires de Louvain, p. 25-41.

ROY, Shirley, et Roch HURTUBISE (2007). L'itinérance en questions, Québec, Presses de l'Université du Québec, 408 p.

SAELINGER, Donald (2006). « Nowhere to Go: The Impacts of City Ordinances Criminalizing Homelessness ", Georgetown Journal on Poverty Law \& Policy, Vol. 13, № 3, p. 545-560.

SERVICE DE POLICE D'OTTAWA (SPO) (2013). Rapport annuel 2013 de la police d'Ottawa : Les partenariats atténuent les nuisances au centre-ville, réf. du 12 avril 2016, http://www.ottawapolice.ca/fr/annual-report/Partnerships-make-a-dent.asp

SERVICE DE POLICE D'OTTAWA (SPO) (2014). Demande d'accès à l'information (14-315) portant sur la Loi de 1999 sur la sécurité dans les rues, [document non publié en possession de l'auteure], Ottawa, Bureau d'accès à l'information de la Police d'Ottawa.

SERVICE DE POLICE D'OTTAWA (SPO) (2015a). Bilan d'une campagne zéro tolérance de cinq jours menée au centre-ville, réf. du 7 octobre 2015,

http://www.ottawapolice.ca/fr/news/index.aspx?newsId=a8500cb8-804a-45fc-92eaf4e78693f1cf

SERVICE DE POLICE D'OTTAWA (SPO) (2015b). Une initiative tolérance zéro menée au centre-ville pendant une semaine au centre-ville se solde par 59 chefs d'accusation criminelle et 338 infractions, réf. du 7 octobre 2015, http://www.ottawapolice.ca/fr/News/index.aspx?newsId=0d4d1d0b-8c5b4600-8d89-af104a6f6ebb 
SEYMOUR, Andrew (2015). "Ticket Defence Program to Fight Back on Behalf of Homeless ", Ottawa Citizen, réf. du 10 avril 2016, http://ottawacitizen.com/news/local-news/ticket-defenceprogram-to-fight-back-on-behalf-of-homeless

SHIP, Susan J. (2001). Rapport final de la consultation communautaire sur les sans-abri autochtones - Région de Montréal, Montréal, Centre d'amitié autochtone de Montréal, 38 p.

SOCIAL DATA RESEARCH LTD. (2005). Evaluation of and Recommendations for Services for the Aboriginal Homeless in Ottawa, [rapport], National Homeless Initiative, Government of Canada, $44 \mathrm{p}$.

SOMERSET WEST COMMUNITY HEALTH CENTRE (2012). Somerset West Community Health Centre, réf. du 29 septembre 2015, http://www.swchc.on.ca

SYLVESTRE, Marie-Ève (2008). La pénalisation et la judiciarisation des personnes itinérantes au Québec: des pratiques coûteuses, inefficaces et contre-productives dans la prévention de l'itinérance et la réinsertion des personnes itinérantes, [mémoire], Québec, Commission parlementaire sur l'itinérance, 38 p.

SYLVESTRE, Marie-Ève, et collab. (2011). «Le droit est aussi une question de visibilité : occupation des espaces publics et parcours judiciaires des personnes itinérantes à Montréal et à Ottawa ", Revue Canadienne Droit et Société, Vol. 26, No 3, p. 531-551.

WACQUANT, Loïc (2012). «La régulation punitive de la pauvreté à l'ère néolibérale ", L'Émancipation syndicale et pédagogique, réf. du 6 avril 2016, http://loicwacquant.net/assets/Papers/REGULATIONPUNITIVEPAUVRETRE.pdf

WAGNER, Vit (2015). «Study calls for repealing Safe Streets Act », Toronto Star, réf. du 20 mars 2015, http://www.thestar.com

WALSH, Christine A., et collab. (2011). «Homelessness and Incarceration among Aboriginal Women: An Integrative Literature Review ", Pimatisiwin: A Journal of Aboriginal and Indigenous Community Health, Vol. 9, No 2, p. 363-386.

WEBSTER, Andrew (2007). Sheltering Urban Aboriginal Homeless People, Assessment of Situation and Needs, [rapport], National Association of Friendship Centres, The Institute of Urban Studies, University of Winnipeg, $180 \mathrm{p}$.

\section{Jurisprudence}

Barreau du Haut-Canada, Code de déontologie, 2014, ch.VI, r 6.3.

Code de la route, LRO 1990, c H.8.

Règlement de la Ville d'Ottawa en matière de contrôle et de soin des animaux (Règlement $\left.N^{\circ} 2003-77\right)$.

Loi sur l'accès à la justice, LO 2006, c 21.

Loi sur la capitale nationale, LRC 1985, c N-4.

Loi sur la protection de l'environnement, LRO 1990, c E.19.

Loi sur la sécurité dans les rues, LO 1999 c 8.

Loi sur le Barreau (1990), LRO 1990, c L8.

Loi sur l'entrée sans autorisation, LRO 1990, C-T21.

Loi sur les permis d'alcool, LRO 1990, L.19.

Loi sur les infractions provinciales 1990, LRO 1990, c P. 33.

Projet de loi 94, Loi Peter Kormos sur l'abrogation de la Loi sur la sécurité dans les rues (2015). 
R. c. Banks 2007, ONCA 19.

Règlement administratif $n^{\circ} 5$ de la Loi sur le Barreau.

Règlement de la Ville d'Ottawa en matière de bruit (Règlement $\left.N^{\circ} 253-2004\right)$.

Règlement sur les propriétés de la Commission de la capitale nationale et la circulation sur ces dernières, CRC, C-1044.

Règlement de la Ville d'Ottawa sur le transport en commun (Règlement N²007-268). 